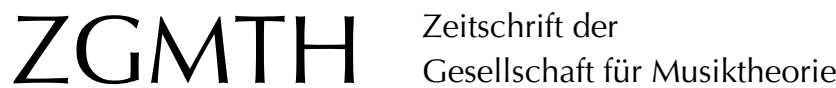

Scheideler, Ullrich (2014): Felix Diergarten, ’edem Ohre klingend. Formprinzipien in Haydns Sinfonieexpositionen, Laaber: Laaber 2012. ZGMTH 11/1, 159-165.

https://doi.org/10.31751/784

(C) 2014 Ullrich Scheideler

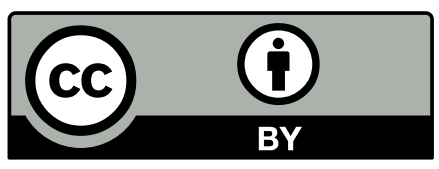

Dieser Text erscheint im Open Access und ist lizenziert unter einer Creative Commons Namensnennung 4.0 International Lizenz.

This is an open access article licensed under a Creative Commons Attribution 4.0 International License.

veröffentlicht / first published: 24/07/2015

zuletzt geändert / last updated: 26/07/2015 


\section{Felix Diergarten, ⿶edem Ohre klingend`. Formprinzipien in Haydns Sinfonieexpositionen, Laaber: Laaber 2012}

In den letzten zwanzig Jahren hat das Interesse an der Sonatenform des 18. Jahrhunderts stark zugenommen. Von amerikanischer Seite haben nach Charles Rosens Sonata forms ${ }^{1}$ dabei vor allem die Untersuchungen von James Webster $^{2}$, Ethan Haimo ${ }^{3}$ sowie die formtheoretischen Entwürfe von William E. Caplin ${ }^{4}$ und James Hepokoski/Warren Darcy ${ }^{5}$, aber auch von Kofi Agawu ${ }^{6}$ neue Impulse gesetzt. Im deutschsprachigen Raum haben sich zuletzt Sonja Gerlach (mit Fokus auf die Chronologie der Sinfonien Haydns ${ }^{7}$ ) und Michael Polth ${ }^{8}$ mit der Frage nach der Funktionsweise der Sonatenform dieser Zeit befasst. Zu Joseph Haydns sinfonischem Schaffen hat jetzt Felix Diergarten eine neue Studie vorgelegt.

Die Einsicht, dass sich ein Sonatensatz um 1760 nicht mit Kategorien der Sonatentheorie des 19. Jahrhunderts (Adolf Bernhard Marx) beschreiben lässt, hat in der Forschung zwei Fragenkomplexe in den Mittelpunkt rücken lassen: den nach der Herkunft, Genese und Entwicklung der Sonatenform sowie den nach den angemessenen Analysekategorien und damit ihren wesentlichen Konstituenten. Als zusammenfassendes Ziel wurde dabei eine Systematisierung der unterschiedlichen $>$ Normen, Typen und Deformationen (so der Untertitel der Publikation von Hepokoski/Darcy) angestrebt, basierend auf der schon von Rosen hervorgehobenen Beobachtung, dass es die Sonatenform (im Singular) nicht gibt, dass

\footnotetext{
1 Rosen 1982.

2 Insbesondere Webster 1991.

3 Haimo 1995.

4 Caplin 2000.

5 Hepokoski/Darcy 2006.

6 Agawu 1991.

7 Gerlach 1996.

8 Polth 2000.
}

sie vielmehr in verschiedenen Formen oder Varianten vorliegt. Dabei ist stets versucht worden, auch eine Entwicklung herauszuarbeiten, die die Genese aus älteren Formmodellen sowie Veränderungen des Modells sichtbar macht. So folgt die Ordnung, wie sie etwa Hepokoski/Darcy vornehmen, in dieser Hinsicht auch einer historischen Einordnung, denn der im Mittelpunkt stehende und am häufigsten anzutreffende Typus ist eben stype $3 \iota^{9}$, der zeitlich später als stype $1 \iota^{10}$ und stype $2 r^{11}$ auftritt (was nicht ausschließt, dass alle Typen auch zeitgleich vorkommen konnten). Ähnlich hatte bereits Polth drei Typen an Expositionen (mit verschiedenen Schemata) beschrieben, die ebenfalls eine chronologische Abfolge implizieren. ${ }^{12}$

9 »Type 3 sonatas are standard textbookı structures, with expositions, developments, and recapitulations that normally begin with $\mathrm{P}$ [primary-theme zone] in the tonic " (Hepokoski/ Darcy 2006, 344, Hervorhebung original).

10 »The pure Type 1 sonata contains no development or extensive elaboration in the second rotation (the recapitulation). Whenever such an elaboration does occur, we may speak of an expanded Type 1 sonata: an expositional rotation followed by an expanded restatement.« (Ebd., 349)

11 »n a Type 2 sonata, however (sometimes thought of as a sbinary variant the exposition may or may not be repeated, and the second rotation begins as a developmental space; only in its second half-often from S onward-does it take on srecapitulatory< characteristics.« (Ebd., 353)

12 »Eine Exposition des dritten Typs setzt voraus, daß die Eröffnungsmusik an einer bestimmten Stelle, die technisch eine Art Halbschluß darstellt, beendet und abgelöst wird von einer sinneren Musik. [...] In den Sinfonien des zweiten Typs existiert zwar ein Teiler, der den 
Wer eine frühe Sinfonie Haydns oder Wagenseils hört, wird schnell feststellen, dass die Kategorien, die aus einer Beethoven-Sinfonie bekannt sind, oft nicht weiterhelfen. Im Zentrum der Analyse muss daher die Frage stehen, wie eigentlich musikalischer Zusammenhang, der als funktionaler Zusammenhang verstanden werden muss, also als Nach- und Miteinander hierarchisch auf einander Bezug nehmender Abschnitte, zustande kommt und wodurch Formbedeutungen entstehen. Als ein wesentlicher Teilaspekt hat sich dabei die Frage herauskristallisiert, inwiefern harmonischen und/oder thematischen Prozessen bei der Konstituierung der Sonatenform die wesentliche Rolle zufällt. Um diese Frage beantworten zu können, sind recht unterschiedliche Strategien verfolgt worden. Hepokoski/Darcy setzten stark auf die Entwicklung einer eigenen Terminologie, die sich an unterschiedlichen Kadenzen und Formfunktionen orientiert, wobei im Mittelpunkt die Idee einer `Rotation`, d.h. einer zirkulären Struktur steht, die im Kontext einer tonalen Ordnung auf einer mehrfachen (variierten) Abfolge desselben musikalischen Materials beruht. Historische musiktheoretische Quellen (u.a. Heinrich Christoph Koch und Georg Joseph Vogler) werden zwar einbezogen, spielen aber keine entscheidende Rolle bei der Konzeption des Buches. Auch Polths Arbeit rekurriert kaum auf historische Zeugnisse, sondern stellt Analysestrategien in den Mittelpunkt, die auf Heinrich Schenker zurückgehen und das Moment der Tonalität ins Zentrum stellen. Dem entgegen stehen Ansätze, die dezidiert die Anschauungen zur Form (oder Gliederung) berücksichtigen, wie sie in den Kompositionslehren nach der Mitte des 18. Jahrhunderts (vor allem bei Joseph Riepel und Koch) zu finden sind (in diese Richtung gehen die Arbeiten von Gerlach und Gjerdingen ${ }^{13}$ ).

Eingang beendet. Doch richtet sich der Formablauf nach der Eröffnungsmusik sogleich auf das Schließen ein. [...] In Expositionen des ersten Typs verhält sich die neutrale Binnenmusik indifferent gegenüber Zuordnungen zu bestimmten Bereichen der Exposition.« (Ebd., 202)
Vor dem Horizont dieser vielschichtigen und durchaus unübersichtlichen Problemstellungen ist nun Diergartens Buch zu besprechen. Wie der Untertitel »Formprinzipien in Haydns Sinfonieexpositionen « andeutet, befasst sich der Autor zwar mit nur einem Komponisten, nimmt dafür aber dessen gesamtes sinfonisches Schaffen, das sich über gut vierzig Jahre (von vermutlich 1757 bis 1795) erstreckt, in den Blick (hinzu kommen einige wenige Seitenblicke auf andere Komponisten - so auf Johann Stamitz -, doch wird etwa Mozart nur ganz am Rande berührt). Diergarten will einen Überblick über die Wandlungen der Sonatenform in Haydns Sinfonik geben, und der Haupttitel "Jedem Ohre klingend" - ein Zitat aus Haydns Oratorium Die Schöpfung - ist ein erster Hinweis darauf, dass für den Autor die Mitte der 1770er-Jahre eine wichtige Station markiert, weil Haydn hier zu einer Ausprägung des Modells gefunden hatte, das danach sowohl im Hinblick auf die Formprinzipien als auch die Themencharaktere nur noch verhältnismäßig geringe Änderungen erfuhr und sich stark am (potentiellen) Publikumsgeschmack orientierte. Diergarten bezieht mithin in seine Argumentation auch soziologische und ästhetische Überlegungen mit ein. Insofern liegt keine rein musiktheoretische oder formanalytische Arbeit vor.

Überhaupt ist der stark synthetische Zug der Arbeit nicht zu übersehen. Neben der Mischung aus systematisierender und musikhistorischer Perspektive wird auch der Versuch unternommen, zwischen den Polen einer Analyse auf Grundlage historischer Quellen und auf Grundlage (partiell) davon unabhängiger neuer Anschauungen zu vermitteln. Diergarten hat die Arbeit daher in

13 Vgl. Gjerdingen 2007. Gjerdingen rekurriert sowohl auf Satz- und Kompositionslehren des 18. Jahrhunderts als auch auf die Partimento-Tradition dieser Zeit. Im Unterschied zur Formbildung in der Sonatenform des späten 18. Jahrhunderts geht er aber für die Phase zwischen ca. 1720 und 1780 davon aus, dass die Satzmodelle (wie Romanesca, Fonte, Monte etc.) in relativ freier Abfolge aufeinander folgen können. 
zwei Teile untergliedert. Der erste Teil (»Zur Genealogie der Formenlehre« [19-47]) dient der Erläuterung der Methode. Diergarten umreißt hier die Ausgangslage und stellt mehrere Punkte heraus. Erstens sei es für die Zeit zwischen ca. 1750 und 1800 schwierig zu bestimmen, was man sich unter dem Formbegriff überhaupt vorzustellen habe, denn es gebe hier weder ein verbindliches Schema noch in Lehrbüchern explizit formulierte Regeln, sondern vor allem die Kompositionen selbst sowie eine Partimentopraxis, an denen implizit Prinzipien der Formbildung abgelesen werden konnten. Daher könne nicht das Verhältnis von Norm und Abweichung im Mittelpunkt stehen (wie im 19. Jahrhundert), sondern müsse sich die Untersuchung auf eine »intratextuelle Ebene« (27), also die unmittelbare Abfolge von Teilen mit ihren Eigenschaften und Besonderheiten konzentrieren. Zweitens benennt Diergarten zwei Konstituenten der Form: die Interpunktion bzw. Endigungsformeln sowie die Themen bzw. den melodischen Inhalt. Im Verlauf des 18. Jahrhunderts komme es zu einer Verschiebung, was an Schriften zeitgenössischer Theoretiker ablesbar sei: So werde etwa bei Koch der melodische Inhalt nur kasuistisch angegeben, während die Abfolge der Endigungsformeln genauer geregelt gewesen sei (bei Joseph Riepel werden allerdings auch Prinzipien der Abfolge von Taktgruppen im Hinblick auf ihren melodischen Inhalt diskutiert $^{14}$ ). Später rücke immer stärker eine Vorstellung von symmetrisch gegliederten Themen in den Vordergrund, die zum einen sogenannte "thematische Routen " 26 und 33 ) in die Sonatenform hineintrügen und zum anderen eine Unterscheidung von Thema und Nicht-Thema ermöglichten. Gewährsmann für den Wandel der Hierarchie der Momente ist für Diergarten Anton Reicha, dessen frühe Schrift Traité de mélodie ${ }^{15}$ sich noch auf Taktgruppen und Endigungsformeln konzentriert, während das spätere Traité de haute composition musicale ${ }^{16}$ das Thema ins Zentrum rückt.

14 Vgl. Riepel 1752, $4 \mathrm{ff}$.

15 Reicha 1814.

16 Reicha 1824/26.
(Die Schriften stammen allerdings somit aus deutlich späterer Zeit, so dass noch genauer untersucht werden müsste, ob hier wirklich eine verspätete Rezeption eines Wandels der Formanschauung auszumachen ist, der in der Musik selbst schon ca. vierzig Jahre früher anzusetzen ist, oder nicht eher eine individuelle Änderung der Perspektive vorliegt.) Da für die Zeit ab ca. 1760 ein Wandel der Formkonzepte angenommen werden müsse, könne nicht a priori eine bestimmte Kategorie als wesentlich angenommen werden, so dass folglich ein Methodenpluralismus sinnvoll sei.

Der zweite und als Hauptteil bezeichnete Teil (»Die Form der Sonatenexposition nach chronologischen Gruppen « [49-185]) gibt in chronologischer Folge einen Überblick über die Formprinzipien der Expositionen in Allegro-Kopfsätzen in Haydns Sinfonien. Dabei folgt jeweils auf einen Abschnitt mit Überblickscharakter die Analyse einer ausgewählten Exposition, so dass am Ende (bei zehn chronologischen Gruppen, von denen die erste geteilt wird) insgesamt elf Einzelanalysen vorliegen (Allegro-Kopfsätze der Sinfonien $1,6,39,26,65,51,53,78,87,89$ und 102). Eingeschaltet sind zwei Exkurse (zur »Monothematik«" [89-116] und zum »Prinzip Wiederholung« [160-164]). Die chronologischen Gruppen hat Diergarten teils der Studie von Gerlach entnommen (für die Sinfonien bis 1774), teils der Werkliste im Haydn-Lexikon. ${ }^{17}$

Die Abschnitte mit Überblickscharakter versuchen eine Ordnung des Materials. Methodenpluralismus wird dabei teils als Zusammenfassung, teils als Wechsel der Analysekategorien verstanden. Das hat zwar den Vorteil, dass sich die Analyse flexibel dem Wechsel der kompositorischen Faktur anpassen kann, hat aber den Nachteil mangelnder Vergleichbarkeit zwischen frühen und späten Werken (so wird etwa bei der Analyse der Exposition des Kopfsatzes der Sinfonie 51 auf die Interpunktion nur ganz sporadisch eingegangen). Erst die Tabellen am Ende geben einen Überblick über alle Sinfonien nach beibehaltenen

17 Raab/Siegert/Steinbeck 2010, 862 ff. (Werkverzeichnis). 
Kriterien. Es überrascht kaum, dass die Reichweite der thematischen Analyse im Sinne einer Sonatenform des 19. Jahrhunderts für die frühen Sinfonieexpositionen äußerst begrenzt ist; dagegen lassen sich bei der Betrachtung der Interpunktion deutlich bestimmte Muster erkennen. Für das frühe Repertoire bemüht Diergarten allerdings Modelle, die er bei Koch findet, um Übereinstimmung und Abweichung zu ermitteln. Die frühen Sinfonien bieten in dieser Beziehung zwar kein ganz einheitliches Bild, weil mehrere Möglichkeiten der Abfolge von Schlusswendungen (Halb- resp. Ganzschluss in I. resp. V. Stufe und Platzierung von Zäsuren) nebeneinander existieren, zeigen aber doch als Ganzes ähnliche Grundrisse. Diergarten kann anschaulich zeigen, wie sich die Möglichkeiten der interpunktischen Route im Laufe seines Schaffens erweitern und von Haydn gezielt auch als Moment der Differenzierung (oder Abwechslung) im Rahmen einer Serie von drei bzw. sechs zusammengehörenden Sinfonien genutzt werden. Hingegen wird die Themenbildung stärker standardisiert, sie folgt seit den 1770er-Jahren deutlicher den aus späterer Zeit bekannten Modellen und tendiert dabei zu symmetrischen Gebilden und motivischen Gestalten. Ausführlichere Überlegungen widmet Diergarten den Anfängen sowie den Kontrastepisoden. Bei den Anfängen kann er zeigen, wie Anfänge im Tutti (so etwa in den Sinfonien 1, 37, 2 etc.) bald Anfängen mit kleiner Besetzung (und im Piano) Platz machen, was Diergarten mit dem Verschwinden des langsamen Kopfsatzes (so noch in den Sinfonien 18, 11, 5) und der langsamen Einleitung (so noch in den Sinfonien $25,6,7)$ in Zusammenhang bringt. (Um diese - einleuchtende - These zu erhärten, wären Untersuchungen zu Sinfonien anderer Komponisten nötig.) Die Kontrastepisoden sind deshalb von Interesse, weil hier ein $\mathrm{Zu}$ sammenhang mit demjenigen vorliegen könnte, was später als 2. Thema bezeichnet wird. Kontrastepisoden sind in Haydns frühen Sinfonien meist kurze Taktgruppen in der Mollvariante, die jedoch erst nach der Etablierung der Oberquinttonart (nach einem Ganzschluss) erklingen und sich daher partiell von späten
Seitensatzthemen unterscheiden, die den Bereich der Oberquinttonart (allerdings nicht immer) eröffnen. Hier vermitteln die Tabellen im Anhang einen guten Eindruck vom Auftreten, allmählichen Verschwinden bzw. Ersetzt-werden dieser Taktgruppen.

Diergarten sieht wesentliche Stationen der Herausbildung von stabilen, für die chronologisch späteren Sinfonien jeweils verbindlichen Prinzipien an mehreren Punkten der Entwicklung von Haydns Sinfonieexpositionen: 1.) 1763 , als es zum Verzicht auf langsame Einleitung bzw. langsamen Eingangssatz mit der Konsequenz kam, wodurch das Thema nicht mehr im Forte beginnen musste. 2.) um 1767, als Haydn nach (mutmaßlich) fast zweijähriger Pause wieder Sinfonien zu schreiben begann, deren Kopfsätze erstmals eine deutlich größere Ausdehnung sowie einen veränderten Affekt zeigen, außerdem die sthematische Route، standardisieren, während die interpunktische Form stärker variieren konnte. 3.) dann vor allem um 1774, als sich ein Verlauf und Themencharaktere etablierten, die weniger das Erhabene als vielmehr das Eingängige und Populare bemühen. Diergarten kann plausibel machen, dass hierfür neben der Musikkritik und der Änderung des musikalischen Umfelds auch eine Veränderung des Adressatenkreises verantwortlich war, da Haydn nun nicht mehr (ausschließlich) für die Kapelle in Esterházy und Eisenstadt komponierte, sondern sich auch dem freien Markt zuwandte. Mit diesen Werken sind nach Diergarten die Weichen für die Zukunft gestellt (vgl. 141, 144, 188): Die nach 1782 entstandenen Sinfonien (immerhin noch knapp 30) werden daher auf nur gut 20 Seiten des Buches behandelt.

Die Einzelanalysen fallen unterschiedlich ausführlich aus (zwischen drei und sieben Seiten). Das Prinzip der Auswahl wechselt dabei: Es werden meist, aber keineswegs immer solche Kopfsatzexpositionen ausgesucht, die jeweils repräsentativ für Prinzipien einer chronologischen Gruppe sind und diese in Fallstudien veranschaulichen und präzisieren. ${ }^{18} \mathrm{Im}$

18 Unter den elf Sinfonien befindet sich nur eine, die auch Haimo analysiert (Sinfonie Nr. 1). 
Falle etwa der Sinfonien 1 oder 39 (der ersten Sinfonie Haydns in einer Molltonart) verhält es sich genau umgekehrt: Die Sinfonien bieten Sonderfälle, weichen somit vom üblichen Standard ab. Diergarten geht meist recht umsichtig und sorgsam abwägend vor, kalkuliert alternative Deutungen oft mit ein, auch wenn die Analysen nicht immer in jedem Detail überzeugend geraten. Hier zeigt sich zudem eine Schwierigkeit der historisch-informierten Musiktheorie. So interpretiert Diergarten beispielsweise die Generalpausen am Beginn des Kopfsatzes von Sinfonie 39 (T. 4/5, T. 11/12) im Sinne eines Zuwachses an Fasslichkeit und beruft sich dabei auf Hans Georg Nägeli (83)..$^{19}$ Und die Terzfallsequenz ab Takt 17 charakterisiert er als einfach und ungebrochen (87), wobei er für das Verfahren, zwischen die thematischen Abschnitte möglichst klare Sequenzen einzuschieben, um die Formfunktion deutlich hervortreten zu lassen, einen Hinweis bei Georg Joseph Vogler bemüht. Beide Autoren schrieben ihre Bemerkungen aber erst nach 1810, und es mutet daher recht zufällig an, wenn Äußerungen herangezogen werden, die gut fünfzig Jahre nach der Komposition der Sinfonie niedergeschrieben wurden, so dass ihnen kaum Beweiskraft für die Argumentation zukommt. ${ }^{20}$ Die Idee, dass die Abschnitte einer Sinfonieexposition stärker

Bei den Tonarten scheint eine möglichst breite Streuung angestrebt worden zu sein: Drei Sinfonien stehen in unterschiedlichen Molltonarten, die übrigen acht Sinfonien decken ein weites Spektrum der Durtonarten ab (das Moment der Tonartencharakteristik, das für die frühen Sinfonien schon aufgrund der unterschiedlichen Besetzung von Bedeutung ist, spielt für die Analysen allerdings eine nur untergeordnete Rolle).

19 Diergartens Analyse der Exposition des Kopfsatzes von Haydns Sinfonie 39 erschien (in teils veränderter Form) auch in: Diergarten 2009, 67-93.

20 Nägelis Bemerkung (1826, 138f.) zu den Generalpausen als Mittel der Fasslichkeit fällt in einem Kapitel zu Carl Philipp Emanuel Bach, wo sie sich in erster Linie auf dessen "Sonaten, Rondos und freye Phantasieen « bezieht. Voglers Bemerkung von dem »Lückenbüsser" in ihrer satztechnischen resp. sthematischen Faktur differenziert werden und dass daher Formfunktionen deutlicher für den Hörer erfahrbar werden, leuchtet zwar ein; zu fragen wäre aber, ob nicht umgekehrt dadurch neue Möglichkeiten für die Darstellung von Affekten bereitlagen, die erst so etwas Ungewöhnliches wie das Verebben mit anschließender Generalpause zuließ (die Pausen wären dann kein Mittel der Klarheit und Fasslichkeit, sondern gerade umgekehrt ein irritierendes Moment, das erst durch die Fasslichkeit des Beginns möglich geworden war). ${ }^{21}$

Bei der Analyse der Exposition des Kopfsatzes von Sinfonie 53 (155ff.) kann Diergarten anschaulich das Ineinander von groß dimensionierter Exposition, motivischer Variation und dem Gedanken der Fasslichkeit klar machen. $\mathrm{Ob}$ man den Anfang wirklich als »melodische Banalität« (156) charakterisieren sollte, hängt auch von der Interpretation seiner Funktion ab: Er bildet einen Kontrast zu der von Erhabenheit geprägten langsamen Einleitung (die Diergarten nicht in seine Argumentation mit einbezieht), zudem wird er u.a. vom Horn gespielt, so dass eine stopical analysis‘ (die Diergarten für Sinfonie 51 auch in formfunktionaler Hinsicht fruchtbar gemacht hat) hier vielleicht zu einer anderen Qualifizierung führen würde. Plausibel aber erscheint die These, dass hier »oberflächliche Eleganz« (158) und Fasslichkeit oder unmittelbare Verständlichkeit zwei Seiten einer Medaille sind.

Die Kategorie der Fasslichkeit ist denn auch eine wesentliche analytische Leitkategorie des Buches, die im "Zusammenfassung und Ausblick« überschriebenen Schlussabschnitt noch einmal thematisiert und dabei mit der Kategorie des Erhabenen in Verbindung gebracht wird, die im Sinne Kochs als das »wirksam Gemachte« (192) verstanden wird. Für eine weitere Diskussion, welches die musikalischen Korrelate zur Idee der Fasslichkeit sind,

stammt aus seinem vermutlich 1811 verfassten System für den Fugenbau (1811, 38).

21 Diergarten deutet später eine in eine ähnliche Richtung gehende Interpretation auch an (2012, 86 und 88f.). 
wäre wahrscheinlich eine umfassendere Untersuchung der Sinfonien Haydns (also auch der Durchführungen und Reprisen sowie der übrigen Sätze) nötig.

Den Abschluss des Buches bilden drei Tabellen, die eine zusammenfassende Übersicht bieten, wodurch ein unmittelbarer Vergleich der Sinfonieexpositionen ermöglicht wird, so dass man die Veränderungen leicht nachvollziehen kann: Tabelle I bietet einen Überblick über die Interpunktion der Exposition aller Sinfonien in Verbindung mit einer knappen Charakterisierung der Abschnitte (als Hauptsatz, Kontrastepisode, Thema etc.). Tabelle II orientiert sich an Hepokoski/Darcys Sonatentypen und bezieht deren Kategorien (wie searly double returns) ein, Tabelle III differenziert nach Eröffnungsformen (für die chronologischen Gruppen 5-10).

Was hätte man sich für dieses Buch noch gewünscht? Zwei Dinge sollen hier nur genannt werden: Erstens wird mir der Forschungsansatz zur Chronologie innerhalb der chronologischen Gruppen von Gerlach etwas zu schnell und ohne Begründung als "problematisch« (17) qualifiziert; denn immerhin existieren einige datierte Autographe. Von daher wäre zumindest eine (knappe) inhaltliche Kritik an der Mischung von quellenkritischen wie musikalischen Kriterien Gerlachs nötig gewesen (irritierend bleibt zudem, dass die Sinfonien innerhalb der chronologischen Gruppen in den Tabellen bei Diergarten in unterschiedli-

\section{Literatur}

Agawu, Kofi (1991), Playing with signs. A Semiotic Interpretation of Classical Music, Princeton NJ: Princeton University Press.

Caplin, William E., (2000), Classical Form: A Theory of Formal Functions for the Instrumental Music of Haydn, Mozart, and Beethoven, Oxford/New York: Oxford University Press. cher Reihenfolge erscheinen). Zweitens bleibt das Verhältnis zu dem in seiner Fragestellung ganz ähnlichen Buch von Polth, auf das sich Diergarten an wenigen Stellen auch bezieht, prekär. Polth hatte Haydns Sinfonien nicht in seine Untersuchung einbezogen, weil »die Werke [...] eine eigene Allegrosatzmusik ausprägen ${ }^{22}$ (auf diesen Passus weist auch Diergarten hin). Da Polth nicht vorgeführt hatte, an welchen Punkten in Haydns Sinfonien sein Ansatz nicht trägt, umgekehrt Diergarten nicht Polths Ansatz einbezieht, der wie gesagt vor allem Tonalität ins Zentrum rückt, sich stark an Schenkers Ideen orientiert, und eine eigene prägnante Terminologie entwickelt (wie z. B. `Neutrale Binnenmusikı, sentfesselte Tonika s), muss offen blieben, ob/wie beide Autoren hier zusammen kommen könnten.

Diergartens Buch eröffnet neue Einblicke und Perspektiven auf Haydns Sinfonieexpositionen, und es ist gerade in dem Überblick, der über alle 106 Sinfonien gegeben wird, von großem Wert. Es stellt zweifellos nicht nur für die Haydn-Forschung, sondern auch für den Diskurs um die Sonatenform im 18. Jahrhundert einen wichtigen Beitrag dar; denn es fragt und erprobt, wie ein angemessenes Verständnis erreicht werden kann und welches die wesentlichen Konstituenten des Formmodells zu dieser Zeit sein könnten. Man darf auf die weitere Diskussion gespannt sein.

Ullrich Scheideler
Diergarten, Felix (2009), »Haydn, Reicha und zwei Pausen: Formprinzipien im 18. Jahrhundert«, in: Joseph Haydn 1732-1809, hrsg. von Sebastian Urmeneit, Berlin: Weidler, 67-93.

Gerlach, Sonja (1996), Joseph Haydns Sinfonien bis 1774. Studien zur Chronologie (= Haydn-Studien 7), München: G. Henle.

22 Polth 2000, 10. 
Gjerdingen, Robert O. (2007), Music in the Galant Style, Oxford/New York: Oxford University Press.

Haimo, Ethan (1995), Haydn's Symphonic Forms. Essays in Compositional Logic, Oxford: Clarendon Press.

Hepokoski, James / Warren Darcy (2006), Elements of Sonata Theory. Norms, Types, and Deformations in the Late-EighteenthCentury Sonata, Oxford/New York: Oxford University Press.

Nägeli, Hans Georg (1826), Vorlesungen über Musik mit Berücksichtigung der Dilettanten, Stuttgart/Tübingen: Cotta.

Polth, Michael (2000), Sinfonieexpositionen des 18. Jahrhunderts. Formbildung und Ästhetik, Kassel u. a.: Bärenreiter.

Raab, Armin / Christine Siegert / Wolfram Steinbeck (Hg.) (2010), Das Haydn-Lexikon, Laaber: Laaber.

Reicha, Antonin (1814), Traité de mélodie: abstraction faite de ses rapports avec I'harmonie; suivi d'un supplément sur l'art d'accompagner la mélodie par l'harmonie, lorsque la première doit être prédominante: Le tout appuyé sur les meilleurs modèles mélodiques gravés sur 77 pl., Paris: Scherff.

- (1824/26), Traité de haute composition musicale, 2 Bde., Paris: Richault.

Riepel, Joseph (1752), De Rhythmopoeia, Oder von der Tactordnung, Regensburg u. a.: Bader.

Rosen, Charles (1980), Sonata forms, New York: W.W. Norten.

Vogler, Georg Joseph [1811], System für den Fugenbau als Einleitung zur harmonischen Gesangverbindungslehre: Nebst 35 Seiten erklärende Notenbeispiele, Offenbach a. M.: André.

Webster, James (1991), Haydn's sFarewelk Symphony and the Idea of Classical Style: Through-composition and Cyclic Integration in his Instrumental Music, Cambridge/New York: Cambridge University Press. 